\title{
The Effect of Lattice Misfit on Deformation Mechanisms at High
} Temperature

\author{
Benedict M.B. Grant ${ }^{1 \mathrm{a}}$, Elisabeth Knoche ${ }^{1 \mathrm{~b}}$, Michael Preuss ${ }^{1 \mathrm{c}}$, \\ Joao Quinta da Fonseca ${ }^{1 \mathrm{~d}}$, Mark R. Daymond ${ }^{2 \mathrm{e}}$ \\ 1. Materials Science Centre, Manchester University, Grosvenor Street, Manchester, Greater \\ Manchester, M1 7HS, UK \\ ${ }^{2}$ Dept. of Mechanical and Materials Engineering, Queen's University, Kingston, K7L 3N6, Canada \\ a.Benedict.Grant@Manchester.ac.uk, b.Elisabeth.Knoche@postgrad.manchester.ac.uk, \\ c.Michael.Preuss@manchester.ac.uk, d. Joao.Fonseca@manchester.ac.uk, \\ e.Daymond@me.queensu.ca
}

Key words: superalloy, load partitioning, neutron diffraction, crystal plasticity

\begin{abstract}
Understanding the relationship between deformation mechanisms and microstructure is essential if one wants to fully exploit the potential of advanced nickel base superalloys and develop future alloys. In the present work, the influence of the lattice misfit between $\gamma$ and $\gamma^{\prime}$ has been studied by means of in-situ loading experiments using neutron diffraction in combination with crystal plasticity modelling on RR1000 and Alloy 720Li. Both alloys were processed to generate three simplified uni-modal $\gamma^{\prime}$ microstructures to allow determination of $\gamma^{\prime}$ responses and experiments were carried out at $750^{\circ} \mathrm{C}$. The results showed that a positive misfit strain increases the level of load partitioning from $\gamma$ to $\gamma^{\prime}$ during plastic deformation introduced by uniaxial tensile loading.
\end{abstract}

\section{Introduction}

High $\gamma^{\prime}$ volume fraction polycrystalline nickel superalloys (approximately 50\% $\gamma^{\prime}$ ) are an important class of materials used by the gas turbine engine industry for disc applications. These alloys have been designed to perform at temperatures as high as $750^{\circ} \mathrm{C}$, which is important for engine efficiency. A sound, fundamental understanding of the active deformation mechanisms is essential to determine the critical microstructural parameters in these alloys. In nickel-base superalloys, $\gamma^{\prime}$ precipitates are coherent with the $\gamma$ matrix with a cube-cube orientation relationship [1]. The unstressed lattice parameter of $\gamma^{\prime}$ is slightly different from that of the $\gamma$ matrix and as a result internal, coherency stresses exist in both the precipitates and matrix. In addition, internal stresses at operation temperatures can be significantly different from those at room temperature. Currently there is no unambiguous understanding of how these coherency stresses might impact the dislocation mobility and therefore affect the materials performance. Neutron and synchrotron x-ray diffraction studies have shown that significant differences in coherency strain can be observed and that the level of misfit is dependent on the aging procedure and cooling rates [2-5].

The magnitude of the internal coherency stresses in these alloys is proportional to the misfit strain which is defined in Equation 1 [6], where $\delta$ is the misfit strain, $a_{\gamma}$ is the lattice parameter of $\gamma$ and $a_{\gamma}$, is the lattice parameter of $\gamma^{\prime}$.

$$
\boldsymbol{\delta}=\frac{2\left(a_{\gamma^{\prime}}-a_{\gamma}\right)}{\left(a_{\gamma^{\prime}}+a_{\gamma}\right)}
$$

Equation 1

It is generally argued that a large misfit strain makes dislocation movement across the matrix/particle interface difficult and hence strengthens the alloy. Principles of alloy design [7] indicate that a large misfit between coherent $\gamma^{\prime}$ precipitates and $\gamma$ matrix is beneficial for low temperature strength. Conversely, a low misfit is generally preferred for high temperature creep resistance. However, it should be remembered that this reflects only empirical knowledge and that there is no consensus on the generality of this relationship and on the physical mechanisms that explain it.

Measurements of $\gamma / \gamma^{\prime}$ misfit strain in RR1000 and Alloy 720Li have shown that Alloy 720Li tends to have a larger misfit than RR1000 [8]. However, it is not clear if small differences in $\gamma / \gamma$ ' lattice misfit as observed in [8] affect the operative deformation mechanisms. To date, most work has 
attempted to relate $\gamma^{\prime}$ size distributions to mechanical properties [9] but without considering misfit. It has also been shown that deformation temperature [10] and precipitate size [8] have very significant impact on the level of load partitioning between $\gamma$ and $\gamma^{\prime}$ during deformation in both Alloy 720Li or RR1000. In this paper, the focus is on the effect of the $\gamma / \gamma^{\prime}$ lattice misfit. The same type of uni-modal $\gamma^{\prime}$ distributions were generated in RR1000 and Alloy 720Li. The differences in misfit strain were measured for each microstructure at test temperature. Subsequently, neutron diffraction was used to monitor, in-situ, the development of load sharing between the two phases during the early stage of plastic deformation.

\section{Method - Materials}

The materials studied were RR1000 and Alloy 720Li, both produced by the powder route. The composition of both alloys can be found in Table 1 .

Table 1: Composition of RR1000 and Alloy 720Li [11] [1]:

\begin{tabular}{|l|l|l|l|l|l|l|l|l|l|l|l|l|l|l|}
\hline & $\mathrm{Ni}$ & $\mathrm{Cr}$ & $\mathrm{Co}$ & $\mathrm{Mo}$ & $\mathrm{Al}$ & $\mathrm{Ti}$ & $\mathrm{W}$ & $\mathrm{Fe}$ & $\mathrm{Mn}$ & $\mathrm{Ta}$ & $\mathrm{Zr}$ & $\mathrm{Hf}$ & $\mathrm{C}$ & $\mathrm{B}$ \\
\hline RR1000 & $\mathrm{Bal}$ & 15 & 18.5 & 5 & 3 & 3.6 & & & & 2 & 0.06 & 0.5 & 0.027 & 0.015 \\
\hline Alloy 720Li & Bal & 15.9 & 14.4 & 2.96 & 2.53 & 4.96 & 1.26 & 0.09 & 0.02 & & & & 0.02 & 0.014 \\
\hline
\end{tabular}

RR1000 is a nickel base superalloy, which was developed by Rolls-Royce plc. and was designed with the aim of achieving a temperature capability of $1000^{\circ} \mathrm{K}\left(727^{\circ} \mathrm{C}\right)$. RR1000 contains high levels of $\mathrm{Al}, \mathrm{Mo}, \mathrm{Cr}$ and $\mathrm{Co}$. This is a consequence of patent restrictions on the use of tungsten and an attempt to increase the contribution of solid solution strengthening [12].

Both alloys have a high volume fraction of $\gamma^{\prime}$ (about 45 vol.\%) and are optimised for strength and fatigue in disc applications. If heat-treated in a conventional manner, they display a tri-modal $\gamma^{\prime}$ arrangement with 1-3 $\mu \mathrm{m}$ intergranular precipitates which are non-coherent; these are the primary precipitates. In addition there are 120-250 nm coherent intragranular secondary precipitates and 20$50 \mathrm{~nm}$ coherent intragranular tertiary precipitates $[9,13]$. Depending on the heat treatment, the relative volume fraction and particle size of the three $\gamma^{\prime}$ families will vary quite significantly.

It is difficult to study $\gamma / \gamma^{\prime}$ load transfer in materials containing a tri-modal $\gamma^{\prime}$ distribution because the extent to which load transfer occurs is dependent on the $\gamma^{\prime}$ particle size [1]. To avoid this difficulty, model uni-modal microstructures were generated using both alloys through a two-part heat treatment. First, all the samples $\left(12 \times 12 \times 80 \mathrm{~mm}^{3}\right.$ blanks $)$ were solution treated above the $\gamma-$ solvus at $1180^{\circ} \mathrm{C}$ for one hour followed by oil quenching. It was found that the high cooling rate generated a uni-modal distribution of around $40 \mathrm{~nm} \gamma^{\prime}$ particles. The second stage was designed to grow the precipitates to the required size by heat treating of the material at $800^{\circ} \mathrm{C}, 925^{\circ} \mathrm{C}$ or $1050^{\circ} \mathrm{C}$ for 1 hour followed by very slow cooling $\left(0.1-1^{\circ} \mathrm{C} / \mathrm{min}\right)$ to avoid the formation of any secondary $\gamma^{\prime}$ during cooling. The resulting microstructures have uni-modal $\gamma^{\prime}$ distributions with a mean particle size of 80,120 and $250 \mathrm{~nm}$, which will be referred to as fine, medium and coarse microstructures. The slow cooling rate should also result in $\gamma$ and $\gamma^{\prime}$ chemical compositions close to equilibrium.

\section{In-situ straining neutron diffraction}

In-situ loading experiments using neutron diffraction were then undertaken on each specimen at $750^{\circ} \mathrm{C}$. The experiments were carried out on ENGIN-X [14], which is part of the neutron spallation facility ISIS at the Rutherford Appleton Laboratory, Didcot, UK. To collect a diffraction pattern of suitable quality, a counting time of 20 minutes was required. Therefore, the loading was performed in a stepped manner. Diffraction data were acquired at pre-determined load steps during which the sample was held at constant load. At high stress levels, this usually resulted in some creep deformation $\left(<5 \times 10^{-5} \mathrm{~s}^{-1}\right)$ during the measurement. For the tensile sample, a standard cylindrical tensile geometry was used with a $6 \mathrm{~mm}$ diameter and M12 threads. The stress rig was placed horizontally on the beam line in order to record diffraction patterns in the loading and transverse direction simultaneously by using the two available detectors. The incident beam is defined by slits and in the present case they were set to $4 \mathrm{~mm}$ (high) $\mathrm{x} 10 \mathrm{~mm}$ (wide). On the receiving side $4 \mathrm{~mm}$ wide collimators were used. 
The diffraction patterns obtained at the different load levels were subsequently analysed using Topas-V4. The aim of this analysis is to extract diffraction peak positions for both $\gamma$ and $\gamma^{\prime}$ with high accuracy, from which the evolution in internal lattice strains can be derived. This analysis is not straightforward because the fundamental diffraction peaks of $\gamma$ and $\gamma^{\prime}$ are very similar. Both phases have similar crystal structures and lattice parameters with the main difference being that $\gamma^{\prime}$ has an ordered structure. This gives rise to two relatively bright superlattice reflections, a consequence the different neutron scattering properties of the different alloying elements. The

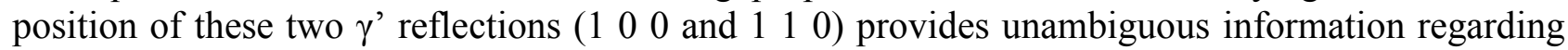
the $\gamma^{\prime}$ lattice strains. All other diffraction peaks are convolutions of diffraction from both phases. In order to determine the lattice strains in the $\gamma$ phase, its contribution to the $\left(\begin{array}{lll}2 & 0 & 0\end{array}\right)$ and $\left(\begin{array}{lll}2 & 2 & 0\end{array}\right)$ peaks must be separated from that of $\gamma$. This was done by using the parameters from the fits to the superlattice peaks to constrain the $\gamma^{\prime}$ parts of the convoluted reflections [15].

\section{EPSC modelling}

In order to interpret the results of the neutron diffraction experiments, elasto-plastic self-consistent (EPSC) modelling was used to calculate the evolution of internal strains during loading [16]. The main model inputs are the single crystal elastic properties, the active slip systems, the critical resolved shear stress required for slip and a hardening law that defines how the slip resistance evolves during deformation. With the exception of the elastic constants, which were assumed to be identical for the two phases in all samples, these parameters were iteratively adjusted until good agreement was obtained with both the macroscopic stress strain curve and the changes in diffraction strain during yielding. Effectively, the diffraction data enable one to constrain the plasticity model and reduce the number of solutions. Deformation was assumed to occur by $(111)<110>$ slip. However, it was found that, in some cases, octahedral slip alone could not give good agreement with the diffraction data. In these cases, the $(100)<110>$ cube slip mode was included in the crystal plasticity model of the $\gamma^{\prime}$ phase. The choice of cube slip is discussed in detail later in the paper.

\section{Results}

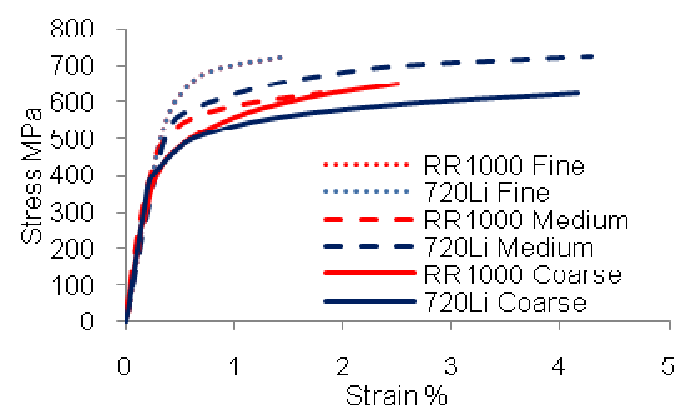

Figure 1: Flow stress curves for all microstructures and for both materials

\section{Mechanical behaviour}

Figure 1 shows the bulk flow stress behaviour for all three microstructures in both materials at $750^{\circ} \mathrm{C}$. It is clear that the yield stress decreases with increased precipitate size. It can be seen that in most situations and for a given $\gamma^{\prime}$ particle size the yield stresses of RR1000 and Alloy $720 \mathrm{Li}$ are very similar. The hardening rate, on the other hand, is significantly different in the two alloys. In Alloy $720 \mathrm{Li}$, the work hardening behaviour is very similar in the different microstructures, whereas it is significantly higher in the coarse RR1000 microstructure.

The greatest difference in yield stress was observed for the "medium" microstructure where the yield stress was approximately $50 \mathrm{MPa}$ higher in Alloy 720Li than in RR1000.

\section{Precipitate size and misfit strain}

Analysis of the diffraction data revealed that the misfit varied with precipitate size. For the (100) oriented grain families and in the case of coarse $\gamma^{\prime}$, the $\gamma / \gamma^{\prime}$ misfit strain was +0.00022 for the Alloy $720 \mathrm{Li}$ and -0.00052 for the RR1000. In the medium microstructure the misfit strain $\gamma / \gamma^{\prime}$ was +0.00019 in Alloy $720 \mathrm{Li}$ and -0.0002 in RR1000. In the case of fine $\gamma$, the misfit strain was +0.0001 for Alloy $720 \mathrm{Li}$ and -0.0001 for RR 1000 . The origin of this link between particle size and misfit strain is not clear. It is possible that it arises from different chemical compositions in the different microstructures, which might have arisen during heat treatment despite the slow cooling rates employed $\left(<1^{\circ} \mathrm{C} / \mathrm{min}\right)$. Another possible explanation could be that as the particle size increases, the contribution of interface energy to the overall equilibrium decreases. This could be compensated by a decrease in the strain energy contribution to reduce the net internal energy. 


\section{Evolution in internal strains during deformation}

Having shown that there is a significant difference in the misfits strain of RR1000 and Alloy 720Li when comparing specific precipitation sizes, it is of interest to determine whether this difference affects the deformation of the alloys by looking at how load partitions between the matrix and the particles during plastic deformation and between the different grain families making up the polycrystals. This is achieved, as described earlier, by "fitting" an EPSC model to the observed macroscopic and diffraction strain responses.

Figure 2 shows an example of diffraction data for RR1000 (a) and Alloy 720Li (b) plotted alongside the diffraction elastic strains given by the EPSC model. It can be seen that the model is successful at capturing the main features of the diffraction data. Of note is the onset of yield and the point at which load is transferred from the $\gamma^{\prime}$ to the matrix. The data also implies that both phases deform
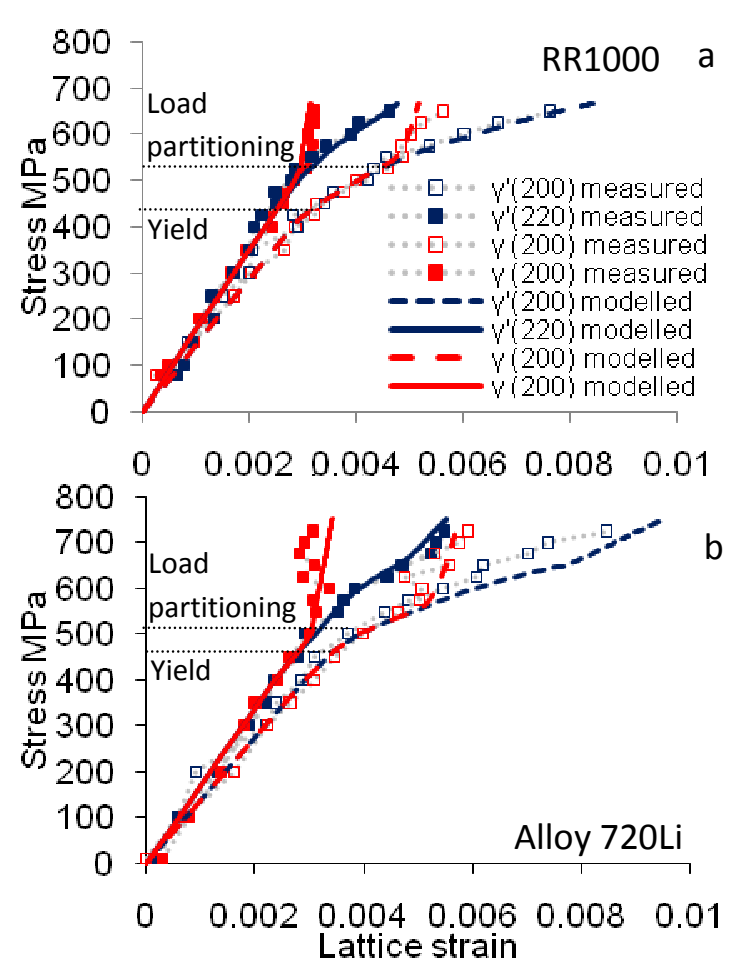

Figure 2: An example of the diffraction results for the medium microstructure for both RR1000 (a) and Alloy $720 \mathrm{Li}(\mathrm{b})$. The markers are the results from diffraction experiments and the lines are the fitted data from the EPSC model plastically. This is suggested by the splitting of the (100) and (110) for the $\gamma^{\prime}$ phase and the (200) and (220) for the $\gamma$ phase. Although there are other possible reasons for this behaviour, the fact that the peak widths increase for both phases seems to corroborate this idea.

However, as it was mentioned earlier, the agreement shown in Figure 2 can only be achieved by introducing (100) slip in the $\gamma$ 'crystal plasticity model, in addition to (111) slip. Some justification for this can be found in the classical idea that cross-slip into the (100) plane occurs in $\gamma^{\prime}$ at high temperatures driven by a decrease in anti-phase boundary energy [17-19]. Whilst there are a number of studies which have shown evidence of (100) slip in single crystal superalloys $[20,21]$, there is only one paper with evidence of (100) slip in polycrystaline superalloys [22]. Furthermore, the difficulty in "fitting" the diffraction data is greatest a low temperature though addition of cube slip does not improve these fits. However, it has been observed in single crystal superalloys that multiple octahedral slip can give rise to shear localisation parallel the (100) plane [23].

The EPSC model does not model dislocation slip explicitly. It only models how intergranular elastic stresses can be relieved by homogenous shear within each grain. Therefore, it is unable to model strain localisation caused by multiple slip. However, if the shear localisation is an important feature of deformation, it will generate intergranular stresses that cannot be predicted by considering only homogenous (111) slip but which will be apparent in the diffraction data. Furthermore, if this localisation has a tendency to occur parallel to the (100) plane, it becomes essentially a pseudo slip system. In an EPSC model, slip on the (100) plane or extensive slip localisation parallel to it are indistinguishable. However such bands should be visible in a post-mortem metallurgical examination where they should appear as slip bands whose trace does not match that of a (111) plane.

\section{Misfit strain and load partitioning}

Previous work on RR1000 has shown that as the precipitate size decreases, onset of load partitioning occurs at increased levels of plastic strain [8]. The diffraction data for Alloy $720 \mathrm{Li}$ shows a similar trend. However, the point at which load partitioning occurs is at lower levels of plastic strain for Alloy 720Li than for RR1000, for the same particle size. 
It could be argued that this implies that the level of plastic strain required for partitioning is directly related to the misfit strain. The positive misfit strain in Alloy $720 \mathrm{Li}$ reduces the level of plasticity required for load partitioning whereas this is delayed in RR1000.

Here EPSC modelling can provide further insight. It shows that although the yield stress is almost identical in the two alloys, the internal strain responses imply that the way deformation is distributed between the two phases is very different in the two alloys, in all three microstructures.

Figure 3 (a) and (b) show the single crystal hardening curves described by the parameters that give the best fit to the experimental data for the medium microstructures. Figure 4 (a) and (b) show how this translates into the amount of plastic strain in each of the phases. They clearly show how, for the same macroscopic strain, the $\gamma$ phase is responsible for a greater share of the total plastic strain in Alloy 720Li than in R1000. The neutron diffraction results suggest that this is a consequence of the positive misfit between $\gamma$ and $\gamma^{\prime}$ in this alloy. So, although the mechanical properties of the two alloys are similar, it appears that at the microstructural scale the partitioning of deformation is quite different. This could have important implications for fatigue resistance, for example, because it implies that, for the same macroscopic strain, the amount of plastic deformation in the matrix is higher in Alloy $720 \mathrm{Li}$ than in RR1000.

Another interesting observation is that the amount of nonoctahedral slip required in $\gamma^{\prime}$ is higher in Alloy $720 \mathrm{Li}$ than in R1000. Should this be a direct consequence of strain localisation, it implies that not only the amount of plastic strain in the $\gamma$ matrix is higher in Alloy 720Li but also that it is more heterogeneous. This would also

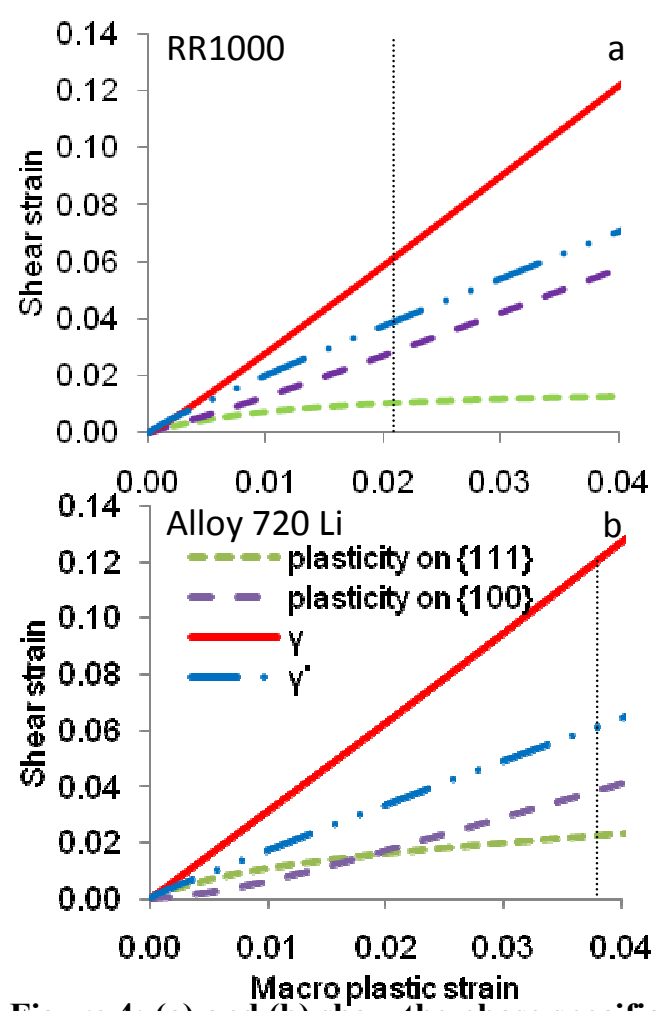

Figure 4: (a) and (b) show the phase specific resolved shear strain and shear strain for each slip mode in $\gamma$ '. The dotted line represents the amount of plastic strain at failure for in-situ loading experiments.
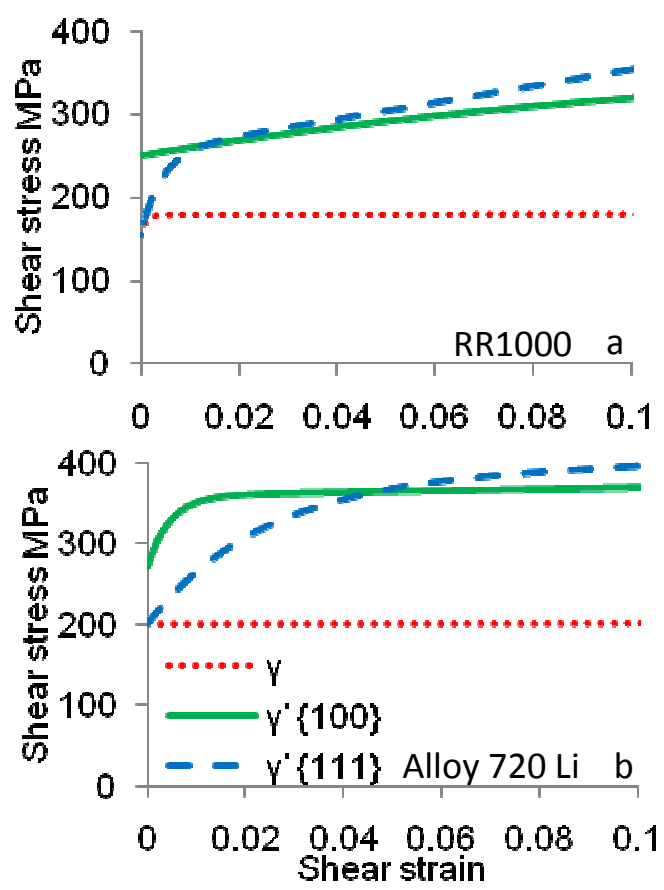
performance performance Figure 3: (a) and (b) show the best fit Voce's because it hardening curves for the EPSC model for implies a high the medium microstructure RR1000 (a) and degree of Alloy 720 Li (b).

localisation and strain reversibility.

It is important to note that some of these results could be explained by differences in the chemical composition between the two materials and therefore different degrees of solid solution strengthening and that the misfit plays a secondary role. However it is clear that the two alloys have significantly different deformation behaviours at the microstructural scale, and that these differences correlate closely with misfit strains.

\section{Conclusion}

This work has confirmed that Alloy $720 \mathrm{Li}$ and R1000 have different $\gamma / \gamma^{\prime}$ misfit strains for the same particle size. Furthermore, measurement of misfit strain indicate that it increases with increasing $\gamma^{\prime}$ particle size.

The results of in-situ straining neutron diffraction experiments, coupled with crystal plasticity modelling have shown that misfit strain correlates well with the onset of load partitioning between $\gamma$ and $\gamma^{\prime}$ particles. 
These results imply that, although the macroscopic deformation behaviour is similar in Alloy $720 \mathrm{Li}$ and RR1000, the local deformation is quite different. The results suggest that, for the same particle size, the amount of plastic deformation is the matrix is higher in Alloy 720Li than in R1000. This result, which has significant implications for the understanding of fatigue performance, has been attributed to the difference in misfit strain in the two alloys.

\section{Acknowledgements}

The authors would like to thank Rolls-Royce plc for supplying material. We would also like to thank the beam line scientist from ENGIN-X, ISIS for their help and support. The project is jointly supported by the EPSRC and MoD (EP/E020933/1).

\section{References}

[1] C.T. Sims, N.S. Stolokoff and W.C. Hagel, John Wiley \& Sons, (1987),

[2] R.J. Mitchell, M. Preuss and M.J. Hardy, Met.Trans. A, 38a, (2007), 615-627

[3] R.J. Mitchell, M. Preuss, M.J. Hardy and S. Tin, Mater. Sci. Eng. A, 423, (2006), 282-291

[4] R.J. Mitchell, M. Preuss, S. Tin and M.C. Hardy, Mater. Sci. Eng. A, 473, (2008), 158-165

[5] J. LI and R.P. WAHI, Acta metall. Mater., 43, 2, (1995), 507-517

[6] R.C. Reed, Cambridge Univeristy Press, (2006),

[7] N. S. Stoloff, John Wiley \& Sons, (1987), 61-96

[8] M. Preuss, J. Quinta da Fonseca, B. Grant, E. Knoche, R. Moat and M. Daymond, (2008),

[9] M. P. Jackson and R. C. Reed, Materials Science and Engineering A, 259, 1, (1999), 85-97

[10] M. R. Daymond, M. Preuss and B. Clausen, Acta Materialia, 55, 9, (2007), 3089-3102

[11] S.J. Hessell, W. Voice, A.W. James, S.A. Blackham, C.J. Small and M.R. Winstone, (1999)

[12] M.C. Hardy, B. Zirbel, G. Shen and R. Shankar, (2004), 83-90

[13] F. Torster, G. Baumeister, J. Albrecht, G. Lutjering, D. Helm and M.A. Daeubler, Mater. Sci. Eng. A, (1997), 189-192

[14] J. R. Santisteban, M. R. Daymond, J. A. James and L. Edwards, Applied Crystalography, 39, (2006), 812-825

[15] H. J. Stone, T. M. Holden and R. C. Reed, Acta Mater, 47, 17, (1999), 4435-4448

[16] B. Clausen, T. Lorentzen, M.A.M. Bourke and M.R. Daymond, Mater. Sci. Eng. A, 259, (1999), 17-24

[17] A.E. Staton-Bevan and R.D. Rawlings, Physica Status Solidi A - Applied Research, 29, (1975), 613-622

[18] J.S. Kohler and F. Seitz, J. Appl. Mech., 14, (1945), A217-A224.

[19] R.J. Taunt and B. Ralph, Philosophical Magazine, 30, (1974), 1379-1394

[20] A. Kostka, G. Malzer, G. Eggeler, A. Dlouhy, S. Reese and T. Mack, J. Mater. Sci., 42, (2007), 3951-3957

[21] G. Eggeler and A. Dlouhy, Acta Mater, 45, 10, (1997), 4251-4262

[22] P.J. Phillips, R.R. Unocic, L. Kovarik, D. Mourer, D. Wei and M.J. Mills, Scripta Mat., 62, 10, (2010), 790-793

[23] D. Bettge and W. Osterle, Scripta Mat., 40, 4, (1999), 389-395 